\title{
Aloperine Inhibits Proliferation and Promotes Apoptosis in Colorectal Cancer Cells by Regulating the circNSUN2/miR-296-5p/STAT3 Pathway
}

This article was published in the following Dove Press journal:

Drug Design, Development and Therapy

\author{
Wei Han ${ }^{1-3, *}$ \\ Desong Kong ${ }^{2, *}$ \\ Qin $\mathrm{Lu}^{4}$ \\ Wei Zhang ${ }^{5}$ \\ Zhimin Fan ${ }^{4}$
}

'Nanjing University of Chinese Medicine, Nanjing, People's Republic of China;

${ }^{2}$ Chinese Medicine Modernization and Big Data Research Center, Nanjing Hospital of Chinese Medicine Affiliated to Nanjing University of Chinese Medicine, Nanjing, People's Republic of China; ${ }^{3}$ General Surgery Department, Nanjing Hospital of Chinese Medicine Affiliated to Nanjing University of Chinese Medicine, Nanjing, People's Republic of China; ${ }^{4}$ Proctology Department, Nanjing Hospital of Chinese Medicine Affiliated to Nanjing University of Chinese Medicine, Nanjing, People's Republic of China; ${ }^{5}$ Anesthesiology Department, Nanjing Hospital of Chinese Medicine Affiliated to Nanjing University of Chinese Medicine, Nanjing, People's Republic of China

*These authors contributed equally to this work

\begin{abstract}
Background: Aloperine can regulate miR-296-5p/Signal Transducer and Activator of Transcription 3 (STAT3) pathway to inhibit the malignant development of colorectal cancer (CRC), but the regulatory mechanism is unclear. This study explored the upstream mechanism of Aloperine in reducing CRC damage from the perspective of the circRNA-miRNAmRNA regulatory network.
\end{abstract}

Methods: After treatment with gradient concentrations of Aloperine $(0.1 \mathrm{mmol} / \mathrm{L}, 0.2$ $\mathrm{mmol} / \mathrm{L}, 0.4 \mathrm{mmol} / \mathrm{L}, 0.8 \mathrm{mmol} / \mathrm{L}$ and $1 \mathrm{mmol} / \mathrm{L}$ ) for 24 hours, changes in CRC cell proliferation and apoptosis were detected by functional experiments. Data of the differential expression of miR-296-5p in CRC patients and healthy people were obtained from Starbase. The effects of Aloperine on 12 differentially expressed circRNAs were detected. The binding of miR-296-5p with NOP2/Sun RNA methyltransferase 2 (circNSUN2) and STAT3 was predicted by TargetScan and confirmed through dual-luciferase experiments. The expressions of circNSUN2, miR-296-5p and STAT3 as well as apoptosis-related genes in CRC cells were detected by qRT-PCR and Western blot as needed. Rescue experiments were conducted to test the regulatory effects of circNSUN2, miR-296-5p and STAT3 on CRC cells.

Results: Aloperine at a concentration gradient inhibited proliferation and promoted apoptosis in CRC cells. The abnormally low expression of miR-296-5p in CRC could be upregulated by Aloperine. Among the differentially expressed circRNAs in CRC, only circNSUN2 not only targets miR-296-5p, but also can be regulated by Aloperine. The up-regulation of circNSUN2 offset the inhibitory effect of Aloperine on cancer cells. The rescue experiments finally confirmed the regulation of circNSUN2/miR-296-5p/STAT3 axis in CRC cells.

Conclusion: By regulating the circNSUN2/miR-296-5p/STAT3 pathway, Aloperine prevents the malignant development of CRC cells.

Keywords: colorectal cancer, aloperine, NOP2/Sun RNA methyltransferase 2, miR-296-5p, signal transducer and activator of transcription 3

\section{Introduction}

Cancer has become the first killer in the world and the biggest obstacle for extending human life expectancy. It is estimated that there were 18.1 million new cancer cases and 9.6 million cancer deaths in $2018 .{ }^{1}$ Colorectal cancer (CRC) is one of the most common gastrointestinal tumors. ${ }^{2}$ According to the data from the International Agency for Research on Cancer (IARC), ${ }^{1}$ the number of new CRC cases worldwide in 2018 was approximately 1.09 million, making CRC the fourth
Proctology Department, Nanjing Hospital of Chinese Medicine Affiliated to Nanjing University of Chinese Medicine, No. 157

Daming Avenue, Qinhuai District, Nanjing City, Jiangsu Province, 210012, People's

Republic of China

Tel +86-255227650।

Email fanzhimin_zhmf@163.com 
most prevalent malignant tumor after lung, breast and prostate cancers; meantime, CRC had a high mortality rate of about $9.2 \%$, ranking only after lung cancer. Epidemiological studies have shown that the incidence of CRC varies significantly in different countries, and is higher in developed areas. ${ }^{3}$ More importantly, as the incidence of CRC rises rapidly in people under 50, the age of onset of the disease is getting lower. ${ }^{4,5}$ Therefore, it is urgent to improve the diagnosis rate and treatment effect of CRC.

Currently, the main clinical treatment of CRC is surgery combined with adjuvant radiotherapy, chemotherapy or molecular targeted therapy. Surgery is generally considered as the first choice for comprehensive treatment of CRC. This method is suitable for patients whose tumors are confined to the intestinal wall while penetrating the intestinal wall and invading the serous or extraserous membrane without lymph node metastasis. ${ }^{6,7}$ However, the availability of radical resection is limited as most patients with CRC have adenocarcinoma, which generally develops from polyps and is metastatic by nature. ${ }^{8}$ As a result, CRC patients tend to undergo simple resection, which leads to a high recurrence rate. Chemotherapy, therefore, is still necessary for postoperative and latestage CRC patients. ${ }^{9,10}$ At present, the commonly used chemotherapeutic drugs in clinic mainly include 5-fluorouracil (5-Fu), oxaliplatin and its derivatives. ${ }^{9}$ Chemotherapy is highly risky because it indiscriminately kills tumor cells and immune cells and thereby reduces the anti-tumor immune effect. ${ }^{11}$ Likewise, radiotherapy greatly weakens the body's immunity. ${ }^{12}$ Hence, researchers have proposed to find effective treatments or drugs that cause less damage to patients.

With advances in the research and development of traditional Chinese medicine, more attention has been paid to the roles of traditional Chinese medicine and its active ingredients in disease prevention and treatment. At the same time, new targets for molecular targeted therapy of diseases have been discovered in the exploration of the molecular mechanisms of traditional Chinese medicine and its monomers. Aloperine (ALO) has been confirmed to have a significant anti-cancer activity. ALO is a component of the traditional Chinese medicine Sophora alopecuroides L. ${ }^{13}$ It is also one of the main alkaloids separated and extracted in the lab, with a molecular formula of $\mathrm{C}_{15} \mathrm{H}_{24} \mathrm{~N}_{2}$. ${ }^{14}$ Recent studies have found that ALO has the effects of anti-inflammation, immunosuppression, redox suppression, cardiovascular protection and anti- cancer, among which its tumor-suppressing activity has been extensively reported. For example, Yu et al revealed that ALO inhibited the carcinogenesis process by regulating excessive autophagy in thyroid cancer cells; ${ }^{15}$ Liu et al demonstrated that ALO inhibited the PI3K/Akt pathway, increased apoptosis and caused cell cycle block in liver cancer cells; ${ }^{16}$ besides, ALO was also found to exert an anti-cancer effect in prostate cancer and breast cancer. ${ }^{17,18}$ In the previous study, our research group found that ALO up-regulated miR-296-5p and inhibited the activity of its target gene STAT3, thereby inhibiting the proliferation and inducing the apoptosis of CRC cells. However, the activation pathway of miR-296-5p is unclear. In order to clarify the upstream activation pathway of ALO-upregulated miR-296-5p, this study will explore the mechanism of ALO in inhibiting CRC from the perspective of the circRNA-miRNA-mRNA regulatory network.

\section{Method \\ Cell Purchase and ALO Processing}

CCD-18Co and CRC cell lines SW480 (CCL-228 ${ }^{\mathrm{TM}}$ ) and HT29 (HTB-38 ${ }^{\mathrm{TM}}$ ) used in this research were provided by the American Type Culture Collection (ATCC). According to the culture requirements, CCD-18Co, SW480 and HT29 cells were separately inoculated in DMEM medium (30-2007, ATCC) containing 10\% fetal bovine serum and incubated in a D180-P cell incubator (RWD, China) containing $5 \% \mathrm{CO}_{2}$ at $37{ }^{\circ} \mathrm{C}$.

ALO (DK0052, CAS NO: 56293-29-9, HPLC $\geq 98 \%$ ), the research object of this study, was provided by Chengdu DESITE Biological Company, China. ALO was dissolved in fresh DMEM to prepare $0.1 \mathrm{mmol} / \mathrm{L}, 0.2 \mathrm{mmol} / \mathrm{L}, 0.4$ $\mathrm{mmol} / \mathrm{L}, 0.8 \mathrm{mmol} / \mathrm{L}$ and $1 \mathrm{mmol} / \mathrm{L}$ ALO solutions, which were separately used to treat the CCD-18Co, SW480 and HT29 cells for 24 hours (h).

\section{MTT Assay}

One hundred $\mu \mathrm{L}$ of SW480 or HT29 cell suspension $\left(1 \times 10^{4}\right.$ cells $\left./ \mathrm{mL}\right)$ was transferred to each well of a 96 well plate. Of note, three holes were added to the same treatment group. After treatment with the ALO solutions for $24 \mathrm{~h}$, SW480 or HT29 cells were added with MTT reagent (10 $\mu \mathrm{L} /$ well, PB180519, Procell, USA), which could react with mitochondria in living cells. After 4 $\mathrm{h}$ of binding, a HBS-1096A enzyme label analyzer (DeTie, China) was used to detect the absorbance of SW480 or HT29 cells in the mixed solutions at $490 \mathrm{~nm}$, 
and then cell viability was calculated based on the absorbance.

\section{EdU (5-Ethynyl-2'-Deoxyuridine) Cell Proliferation Experiment}

SW480 or HT29 cells in logarithmic growth phase were seeded in 96 -well plates at $1 \times 10^{4}$ cells/well. EdU reagent (ST067) purchased from Beyotime was diluted to 50 $\mu \mathrm{mol} / \mathrm{L}$ with fresh DMEM medium. The EdU dilution was then used to incubate the cells at $100 \mu \mathrm{L} /$ well for $2 \mathrm{~h}$. After removing the culture medium, the SW480 or HT29 cells were fixed with methanol at room temperature for 15 minutes (min). Positive fluorescence expression (red) in the collected SW480 or HT29 cells were observed, photographed and recorded under a Leica DMi8 inverted fluorescence microscope (magnification $100 \times$ ). Then DAPI reagent was used to stain the nuclei, and the stains were observed under a microscope.

\section{Colony Formation Assay}

Two hundred SW480 or HT29 cells were transferred to petri dishes containing complete medium $(10 \mathrm{~mL})$. In order to distribute the cells evenly, the petri dishes were rotated slowly for $1 \mathrm{~min}$ after cell inoculation. Next, the culture dishes containing cells were placed in an incubator for routine culture. During the culture, the medium was changed every 2 days (d). After 14 d, the SW480 or HT29 cells were soaked with Giemsa dye for $20 \mathrm{~min}$ and then the colony formation of the cells was observed under a microscope.

\section{Apoptosis Experiment}

An Annexin V-FITC Apoptosis Detection Kit (CA1020, Solarbio, China) and a flow cytometer (CytoFLEX, BECKMAN COULTER, USA) were used to detect changes in the apoptosis of SW480 or HT29 cells. In brief, SW480 or HT29 cells were prepared into $1 \times 10^{6}$ cells/mL cell suspension with $1 \mathrm{~mL} 1 \times$ Binding Buffer. One hundred $\mu \mathrm{L}$ of SW480 or HT29 cells and $5 \mu \mathrm{L}$ of Annexin V-FITC reagent were added to a centrifuge tube and mixed for $10 \mathrm{~min}$ in the dark at room temperature. Next, $5 \mu \mathrm{L}$ of PI reagent was added to the centrifuge tube and incubated in the dark at room temperature for $5 \mathrm{~min}$. Afterwards, PBS was added to adjust the mixture to a final volume of $500 \mu \mathrm{L}$. After half an hour, the cells in the centrifuge tube were transferred to the detection instrument to calculate the number of apoptotic cells.

\section{Bioinformatics Analysis and Target Gene Binding Verification}

Starbase (http://starbase.sysu.edu.cn/index.php) was used to retrieve and analyze information of differential expression of miR-296-5p in Colon adenocarcinoma (COAD) patients and healthy volunteers. TargetScan (http:/www.targetscan.org) was used to predict the circRNA that contained a binding sequence for miR-296-5p and the downstream target genes of miR-296-5p. The binding sequences predicted by TargetScan were designed by COBIOER (China) and used to construct the following reporter plasmids for dual luciferase experiments: NOP2/Sun RNA methyltransferase 2 wild type (circNSUN2-WT), circNSUN2-mutant type (MUT), Signal Transducer and Activator of Transcription 3 (STAT3)-WT and STAT3-MUT. The reporter plasmids were separately cotransfected with miR-296-5p mimic or mimic control into SW480 and HT29 cells using liposome for $48 \mathrm{~h}$. Luciferase activity in the transfected SW480 and HT29 cells was detected using Dual-Luciferase ${ }^{\circledR}$ Reporter Assay System (E1960, Promega, USA) with a GloMax 20/20 luminometer (Promega, USA).

\section{Quantitative Real-Time Polymerase Chain Reaction (qRT-PCR)}

qRT-PCR is a reliable method for rapid detection of gene mRNA levels. Briefly, total RNA of SW480 and HT29 cells was fully isolated by the conventional RNA extraction reagent TRIzol (15,596,018, Invitrogen, USA), and subsequently reverse transcribed into cDNA using the RNA reverse transcription reagent (RR047A) developed by TaKaRa (Japan). The qRT-PCR reaction system which consisted of cDNA, gene primers (Sangon synthesis), SYBR ${ }^{\circledR}$ Green (S4438-20RXN, Sigma-Aldrich, Germany) and DEPC water was added to a 96-well plate and put into the detection instrument (Bio-Rad thermal cycler T100). The reaction conditions were set as follows: pre-denaturation at $95^{\circ} \mathrm{C}$ for $10 \mathrm{~min}$, denaturation at $95{ }^{\circ} \mathrm{C}$ for 15 seconds (s), and annealing at $58{ }^{\circ} \mathrm{C}$ for $1 \mathrm{~min}$, for a total of 40 cycles. According to Anita Ciesielska's report, ${ }^{19}$ gene mRNA levels were quantified by the $2^{-\Delta \Delta C T}$ method. GAPDH and U6 were internal references in the experiment. The specific sequences of synthetic primers were shown in Table 1.

\section{Cell Transfection}

Exogenous up-regulation or knockdown of test genes is a common method to observe their regulatory effects on cells or tissues. Liposome transfection with Lipofectamine 
Table I Primers for qRT-PCR

\begin{tabular}{|c|c|c|}
\hline Gene & Forward Primer (5'-3') & Reverse Primer (5'-3') \\
\hline miR-296-5p & CCTGTGTCGTATCCAGTGCAA & GTCGTATCCAGTGCGTGTCG \\
\hline $\operatorname{circDD} \times 17$ & TGCCAACCACAACATCCTCCA & CGCTCCCCAGGATTACCAAAT \\
\hline circHIPK3 & TATGTTGGTGGATCCTGTTCGGCA & TGGTGGGTAGACCAAGACTTGTGA \\
\hline circZFR & AACCACCACAGATTCACTAT & AACCACCACAGATTCACTAT \\
\hline circRNA_100290 & GTCATTCCCTCTTTAATGGTG & CAGAACTTCCGCTCTAACATAC \\
\hline Has_circ_0026344 & CTCAGCCTCTAGCATAAGCTC & AGGCAAGAGAATGATTTGAAC \\
\hline circVAPA & TGGATTCCAAATTGAGATGCGTATT & CACTTTTCTATCCGATGGATTTCGC \\
\hline Hsa_circ_000936I & AGAACCAGATTCGAGACGCC & GTGCTCTTCAATGCCACCTTC \\
\hline circNSUN2 & CTTGAGAAAATCGCCACACTT & GTTGAGGAGCAGTGGTGG \\
\hline $\operatorname{circACAP2}$ & TCAGAGCATCTGCCCAAAGTT & AAATGAACCCCAAGGCTCCG \\
\hline circlTGA7 & GTGTGCACAGGTCCTTCCAA & TGGAAGTTCTGTGAGGGACG \\
\hline circEIF4G3 & ССТАССССАТССССТТАТТС & ACCGTGCTGTAGACTGCTGAG \\
\hline STAT3 & CCCCATACCTGAAGACCAAG & GGACTCAAACTGCCCTCCT \\
\hline U6 & CTCGCTTCGGCAGCACA & AACGCTTCACGAATTTGCGT \\
\hline GAPDH & TGTGGGCATCAATGGATTTGG & ACACCATGTATTCCGGGTCAAT \\
\hline
\end{tabular}

3000 (L3000008, ThermoFisher Scientific, USA) is the most common and convenient method for exogenous interference. Therefore, we commissioned Guangzhou GENESEED Biological Company to synthesize circNSUN2 overexpressed plasmid, circNSUN2 silenced plasmid (shcircNSUN2), STAT3 overexpressed plasmid and their respective negative controls. MiR-296-5p mimic (miR10000690-1-5) and mimic control (miR1N00000011-5), as well as miR-296-5p inhibitor (miR20000690-1-5) and inhibitor control (miR2N0000001-1-5), were purchased directly from Guangzhou RIOBOBIO Company. The plasmids were transfected into SW480 and HT29 cells according to the grouping using Lipofectamine 3000. After $48 \mathrm{~h}$, the success rate of transfection of the cells was determined by qRT-PCR.

\section{Western Blot}

RIPA lysate (tissue/cell, R0010) produced by Solarbio (China) was used to separate protein from SW480 and HT29 cells, and its concentration was determined with BCA protein concentration determination reagent (PC0020, Solarbio, China). Then the protein was subjected to high temperature denaturation to maintain a relatively stable state. After being electrophoresed by SDS-PAGE, the protein was transferred to the membrane carrier (PVDF membrane). The membrane was soaked with the same BSA sealant (SW3015) produced by Solarbio for $2 \mathrm{~h}$ at room temperature. Next, the membrane was incubated with Abcam (USA) antibodies (anti-STAT3, ab68153, 88 kDa; Bax, ab32503, 21 $\mathrm{kDa}$; Bcl-2, ab59348, $26 \mathrm{kDa}$ ) that could bind to the corresponding antigens in the protein on the membrane overnight $\left(4{ }^{\circ} \mathrm{C}\right)$, with GAPDH $(\mathrm{ab} 8245,36 \mathrm{KD})$ as an internal reference. To identify the antigen-antibody complex, a secondary antibody labeled with HRP (Goat Anti-Rabbit antibody, 1:10,000, ab6721; Goat Anti-Mouse antibody, 1:10000, ab205719) was used to treat the PVDF membrane for $1.5 \mathrm{~h}$ at room temperature. Fluorescent signal of the complex was enhanced using the ECL Western Blotting Substrate (PE0010, Solarbio) and detected using the Gel Doc $^{\mathrm{TM}} \mathrm{XR}+$ imaging system (BIO-RAD, USA). The gray value of the bands was calculated by Image Lab software to determine the final gene protein level.

\section{Statistical Analysis}

SPSS 20.0 software (IBM, USA) was employed for statistical analysis of the data obtained in the experiments. Differences between two groups were compared by independent sample $t$-test, and those between multiple groups were compared by one-way ANOVA followed by Tukey's test. When $p<0.05$, the difference was considered statistically significant.

\section{Result \\ ALO at a Concentration Gradient Inhibited Proliferation Yet Promoted Apoptosis in CRC Cells}

In this study, the toxicity of ALO in normal cells (CCD$18 \mathrm{Co}$ ) was detected, ALO showed no significant toxicity to CCD-18Co cells (Figure 1A). In order to study the effects of ALO on the growth and basic physiological functions of 
CRC cells, we used different concentrations of ALO to treat SW480 and HT29 cells. The results showed that $0.2 \mathrm{mmol} /$ $\mathrm{L}, 0.4 \mathrm{mmol} / \mathrm{L}, 0.8 \mathrm{mmol} / \mathrm{L}$ and $1 \mathrm{mmol} / \mathrm{L}$ ALO solutions all inhibited the viability of SW480 and HT29 cells $(p<0.05$, Figure 1B and C). Moreover, the viability of SW480 and HT29 cells was close to $50 \%$ under the treatment of 0.8 $\mathrm{mmol} / \mathrm{L}$ ALO solution, but was reduced to less than $50 \%$ when the ALO concentration was increased to $1 \mathrm{mmol} / \mathrm{L}$ (Figure 1B and C). In the following EdU cell proliferation experiment, the red fluorescence expression was reduced in SW480 and HT29 cells under the treatment of $0.8 \mathrm{mmol} / \mathrm{L}$ ALO (Figure 1D and E). Consistently, the clone formation experiment also showed that different concentrations of ALO $(0.2 \mathrm{mmol} / \mathrm{L}, 0.4 \mathrm{mmol} / \mathrm{L}, 0.8 \mathrm{mmol} / \mathrm{L})$ reduced the number of cell clones and inhibited the proliferation of SW480 and HT29 cells $(p<0.05$, Figure $1 \mathrm{~F}-\mathrm{H})$. At the same time, the increase in the concentration of the ALO solution accelerated the death of CRC cells. As shown in Figure 1I-K, the number of apoptotic SW480 and HT29 cells increased significantly after treatment with different concentrations of ALO $(p<0.01$, Figure $1 \mathrm{I}-\mathrm{K})$.

\section{The Abnormally Low Expression of miR-296-5p in Colon Cancer Could Be Up-Regulated by ALO}

Data from the Starbase database showed that the expression of miR-296-5p in colon cancer patients was significantly lower than that in healthy people ( $p=2.5 \mathrm{e}-6$, Figure $2 \mathrm{~A}$ ). In this study, however, after treatment with different concentrations of ALO for 24 h, miR-296-5p activity in SW480 and HT29 cells was significantly increased $(p<0.01$, Figure 2B and $\mathrm{C}$ ), suggesting that ALO can stimulate the up-regulation of miR-296-5p.

\section{Among the Differentially Expressed circRNAs in CRC, Only circNSUN2 Not Only Had a Target Site for miR-296-5p, but Also Could Be Regulated by ALO}

Through literature review, we screened 12 circRNAs that were reported to be associated with CRC: circDDX17, circHIPK3, circZFR, circRNA_100290, Has_circ_0026344, circVAPA, Hsa_circ_0009361, circNSUN2, circACAP2, circACVRL1, circITGA7 and circEIF4G3. We first detected the regulatory effect of ALO on these differentially expressed circRNAs by qRT-PCR to perform preliminary screening (Figure 3A). The results showed that the expressions of
circDDX17, Has_circ_0026344, circVAPA, circNSUN2, circACAP2, circACVRL1 and circEIF4G3 were regulated by $0.8 \mathrm{mmol} / \mathrm{L}$ ALO ( $p<0.001$, Figure $3 \mathrm{~A})$. Through subsequent screening of target genes, we found that only circNSUN2 and miR-296-5p have targeted binding sites (Figure 3B). After further verification, it was confirmed that circNSUN2-WT and miR-296-5p mimic did reduce the luciferase activity in SW480 and HT29 cells ( $p<0.001$, Figure $3 \mathrm{C}$ and D). Therefore, we chose circNSUN2 as the next research object.

\section{Overexpression of circNSUN2 Partially Offset the Suppression of ALO on the Biological Behavior of Cancer Cells}

In SW480 and HT29 cells, ALO suppressed the mRNA level of circNSUN2, whereas transfection of circNSUN2 overexpression significantly increased the expression of circNSUN2 $(p<0.001$, Figure 4A and B). The following basic cell function experiments showed that the upregulation of circNSUN2 partially offset the inhibitory effect of ALO on CRC cell viability, proliferation and apoptosis $(p<0.001$, Figure 4C-L).

\section{Sh-circNSUN2 Inhibited the Malignant Development of CRC Cells, Which Was Neutralized by miR-296-5p Inhibitor}

We also tested the mutual regulation of circNSUN2 and miR-296-5p in SW480 and HT29 cells. Sh-circNSUN2 could down-regulate the expression of circNSUN2, and besides, it inhibited the proliferation of SW480 and HT29 cells, while promoting their apoptosis and the expression of miR-296-5p $(p<0.001$, Figure 5A-J). However, the inhibitory effect of sh-circNSUN2 on the malignant development of the two cancer cells was neutralized by miR-296-5p inhibitor $(p<0.001$, Figure $5 \mathrm{~A}-\mathrm{J})$.

\section{MiR-296-5p Bound to STAT3 and Upregulation of Mir-296-5p Inhibited the Expression of STAT3 in CRC Cells}

We screened the target genes of miR-296-5p, and found that miR-296-5p and STAT3 have targeted binding sites (Figure 6A), and this binding relation was further confirmed by a dual-luciferase verification test $(p<0.001$, Figure $6 \mathrm{~B}$ and C). Next, we used qRT-PCR and Western blot to analyze the mRNA and protein levels of miR-296-5p and STAT. It was found that miR-296-5p mimic up-regulated miR-296-5p expression while preventing the activation of STAT3 


\section{A}

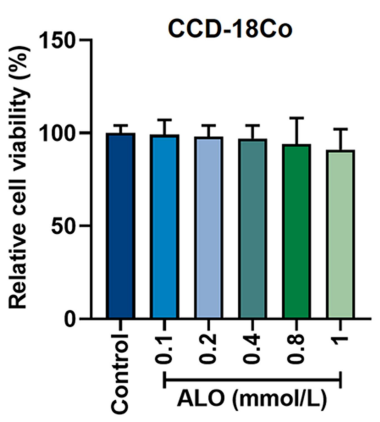

D

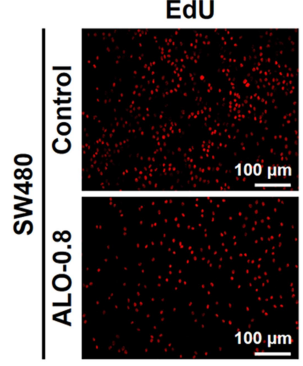

F

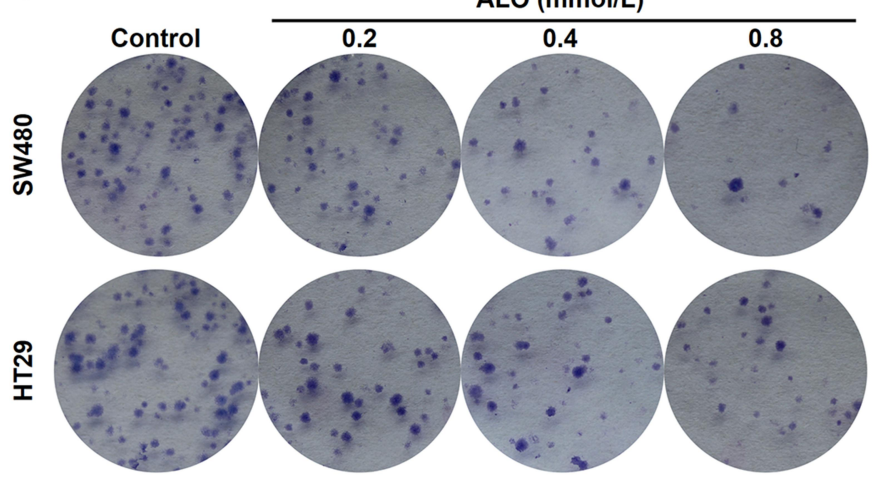

I
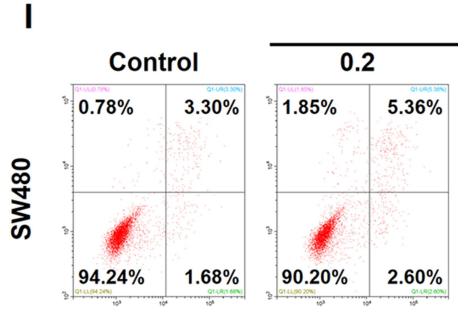

ALO (mmol/L)
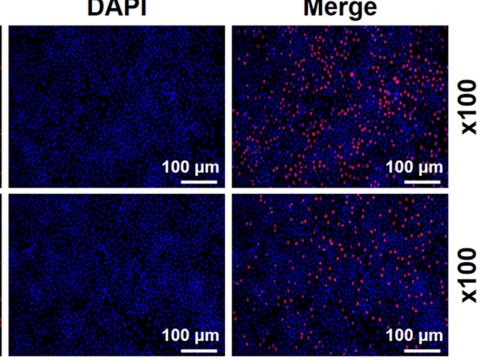

ALO (mmO/L)

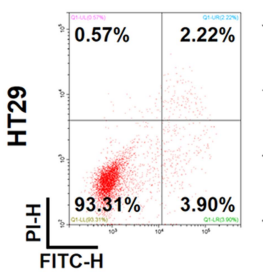

B

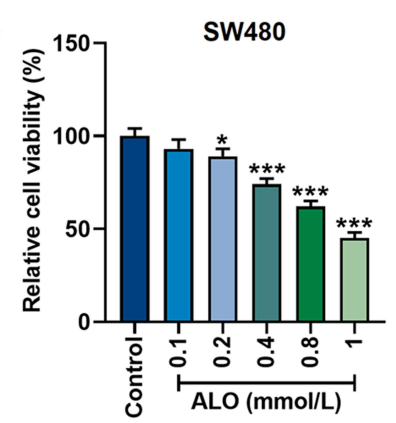

C

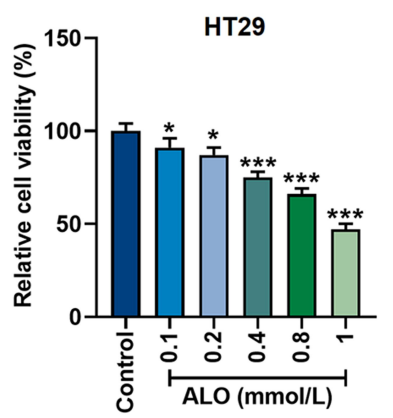

E

EdU

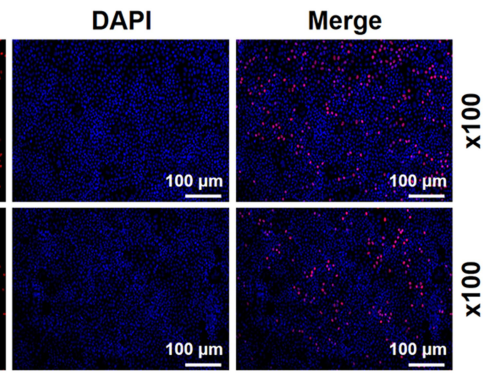

H

G
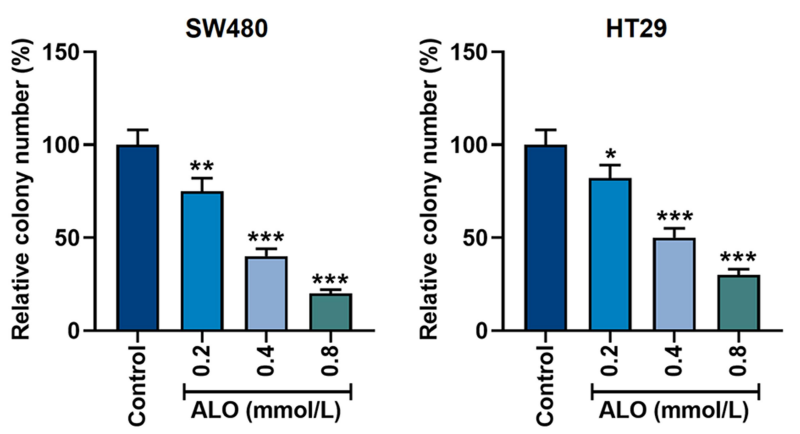

J

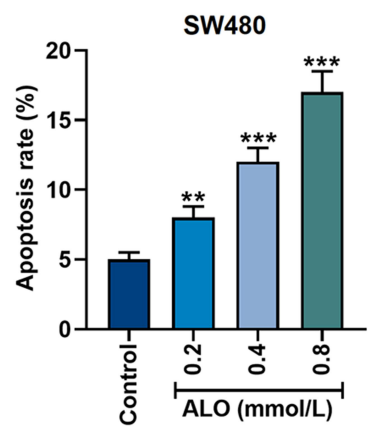

K

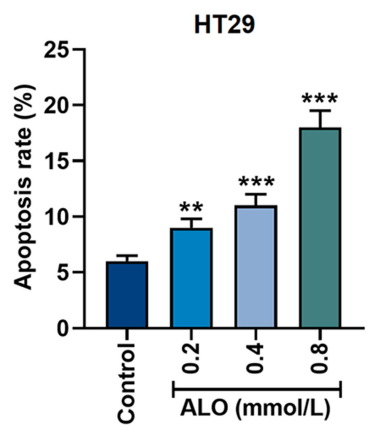

Figure I ALO at a concentration gradient inhibited proliferation yet promoted apoptosis in CRC cells. (A) The effect of ALO on the normal colonic tissue cells (CCD-I8Co) was detected by MTTexperiments. (B and C) MTTexperiments showed that ALO at a concentration gradient $(0.1 \mathrm{mmol} / \mathrm{L}, 0.2 \mathrm{mmol} / \mathrm{L}, 0.4 \mathrm{mmol} / \mathrm{L}, 0.8 \mathrm{mmol} / \mathrm{L}$ and I mmol/L) inhibited the activity of SW480 and HT29 cells. (D and E) EdU cell proliferation experiments showed that $0.8 \mathrm{mmol} / \mathrm{L}$ ALO inhibited the proliferation of SW480 and HT29 cells. The magnification was $100 \times$. (F-H) The clone formation experiment showed that $A L O$ at a concentration gradient $(0.2 \mathrm{mmol} / \mathrm{L}, 0.4 \mathrm{mmol} / \mathrm{L}$ and $0.8 \mathrm{mmol} / \mathrm{L})$ inhibited the proliferation of SW480 and HT29 cells. (I-K) Flow cytometry experiments showed that ALO at a concentration gradient $(0.2 \mathrm{mmol} / \mathrm{L}, 0.4 \mathrm{mmol} / \mathrm{L}$ and $0.8 \mathrm{mmol} / \mathrm{L})$ promoted the apoptosis of SW480 and HT29 cells. All experiments were repeated three times to obtain average values. $*_{p}<0.05$, $* * p<0.01$, $*_{* *}<<0.001$ vs Control.

Abbreviations: ALO, aloperine; CRC, colorectal cancer. 
A

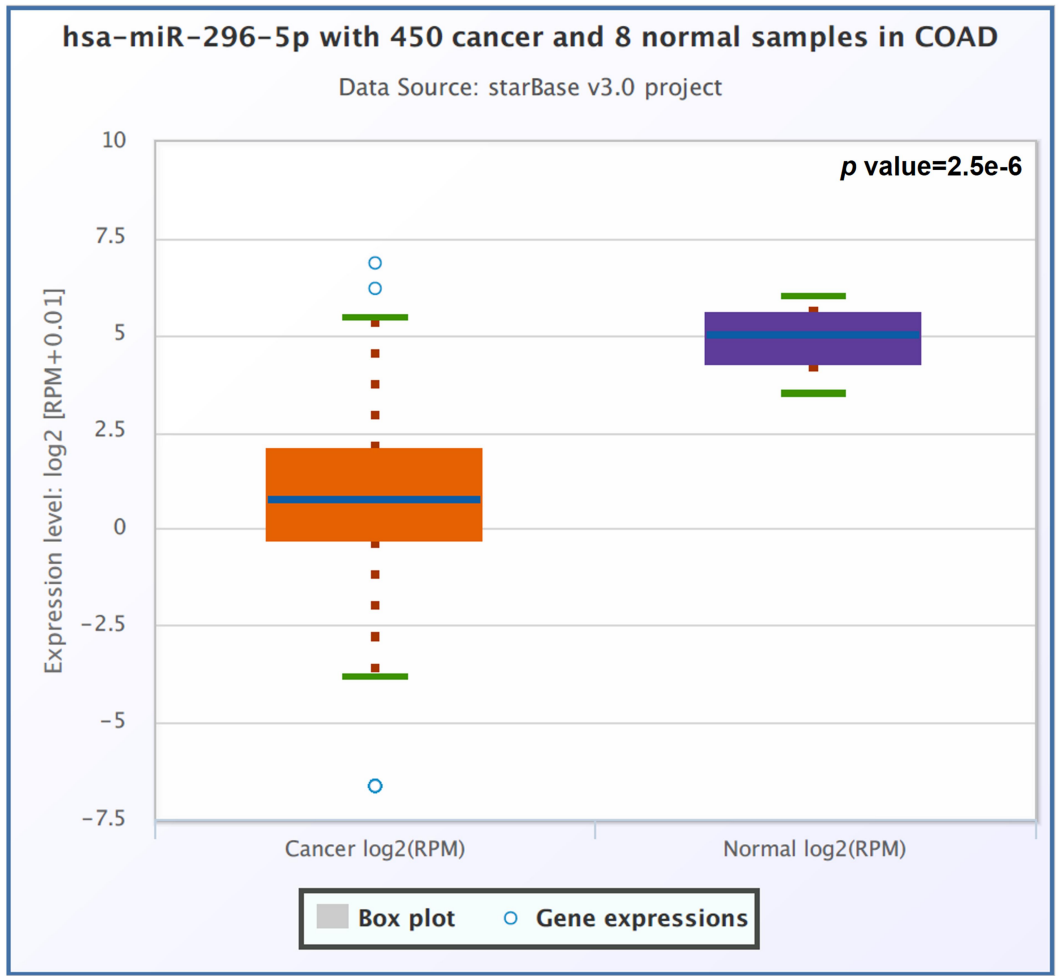

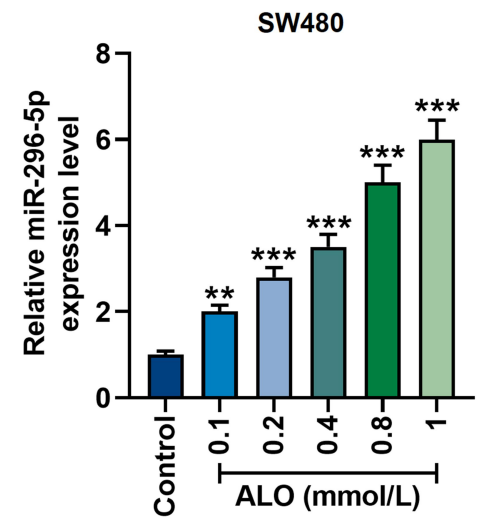

C

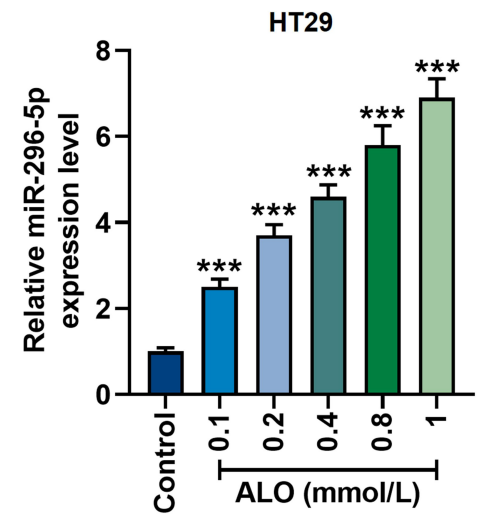

Figure 2 The abnormally low expression of miR-296-5p in colon cancer could be upregulated by ALO. (A) Starbase (http://starbase.sysu.edu.cn/index.php) was used to retrieve the information of differential expression of miR-296-5p in colon adenocarcinoma (COAD, $n=450)$ patients and healthy volunteers $(n=8)$ in vivo. $P=2.5 e-6$. $(B$ and C) The qRT-PCR experiment showed that ALO at a concentration gradient $(0.1 \mathrm{mmol} / \mathrm{L}, 0.2 \mathrm{mmol} / \mathrm{L}, 0.4 \mathrm{mmol} / \mathrm{L}, 0.8 \mathrm{mmol} / \mathrm{L}$ and I mmol/L) up-regulated miR-296-5p expression in SW480 and HT29 cells. All experiments were repeated three times to obtain average values. $* * p<0.01$, $* * * p<0.001$ vs Control.

Abbreviation: qRT-PCR, quantitative real-time polymerase chain reaction.

$(p<0.001$, Figure $7 \mathrm{~A}-\mathrm{G})$. However, the overexpression of STAT3 reversed the regulation of miR-296-5p mimic on the two genes $(p<0.001$, Figure 7A-G).

\section{MiR-296-5p Mimic Inhibited Proliferation and Promoted Apoptosis in CRC Cells by Regulating Apoptosis-Related Genes, Which Was Partially Reversed by Overexpressed STAT3}

The following cell experiments showed that by activating Bax and blocking the protein activity of Bcl-2, miR296-5p mimic inhibited proliferation yet accelerated apoptosis in cancer cells $(p<0.001$, Figure $8 \mathrm{~A}-\mathrm{I})$. However, up-regulation of STAT3 produced a completely opposite regulatory effect $(p<0.001$, Figure 8A-I). More importantly, overexpression of STAT3 neutralized the regulation of miR-296-5p mimic on CRC cells $(p<0.001$, Figure $8 \mathrm{~A}-\mathrm{I})$.

\section{Discussion}

$\mathrm{CRC}$ is a malignant lesion of the mucosal epithelium of the colon or rectum under the action of various carcinogenic factors such as environment or heredity. ${ }^{2}$ Most CRC patients suffer from adenocarcinoma, which usually develops from polyps. Tumors developed from polyps can infiltrate in a circular shape along the horizontal axis of the intestinal tube, develop into the deep layer of the intestinal wall, and finally penetrate the intestinal wall and metastasize to blood vessels or lymphatic vessels. ${ }^{20}$ This is one of the important reasons for the high recurrence and metastasis rates of CRC. The ultimate goals of CRC research are to improve the current treatment of CRC, save patients' lives and improve their quality of life. Based on the role of traditional Chinese medicine in disease prevention and treatment, this study explored the effects of ALO, an alkaloid exhibiting strong anticancer activity, on the proliferation and apoptosis of CRC cells. The results showed that after ALO treatment, the viability and proliferation of 

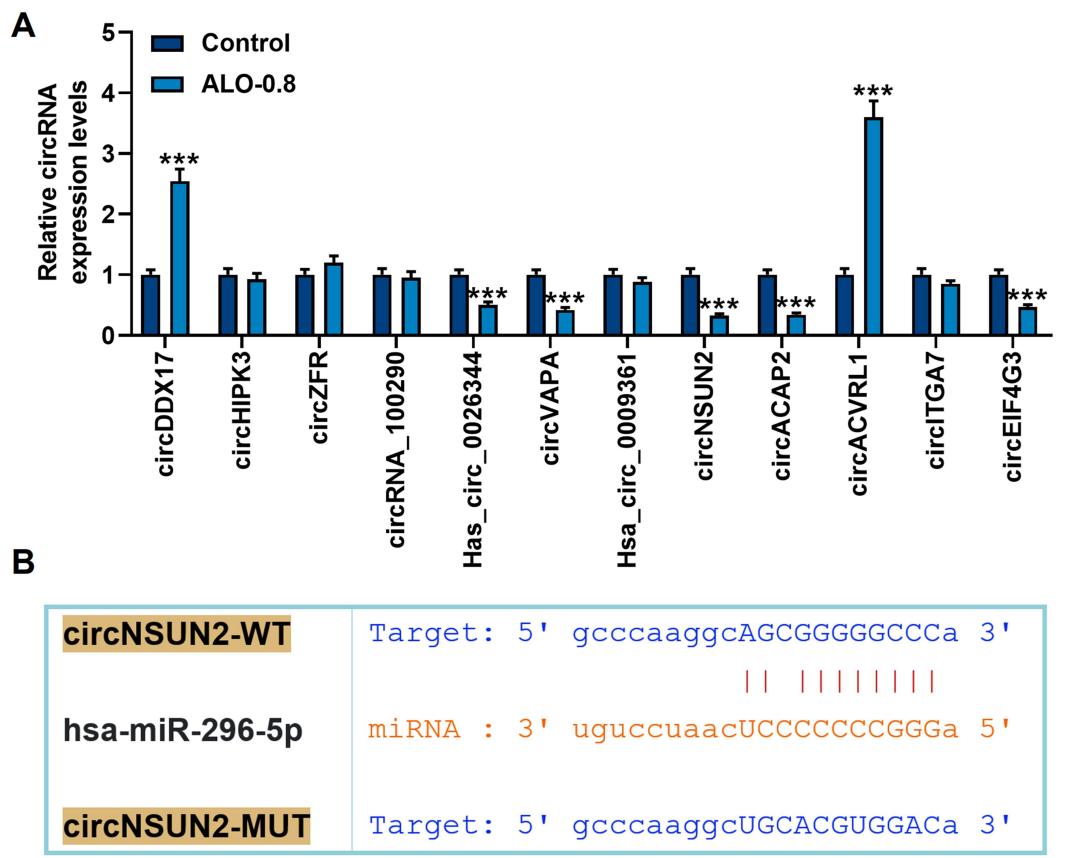

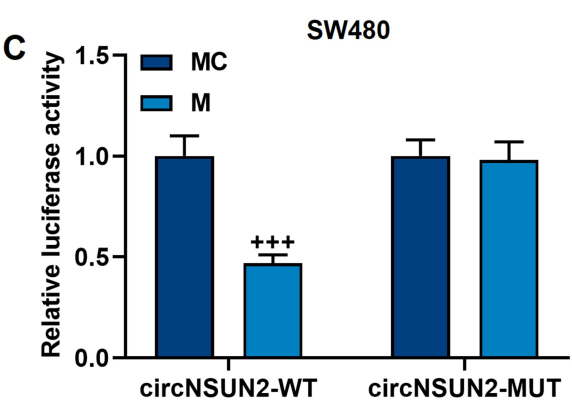

D

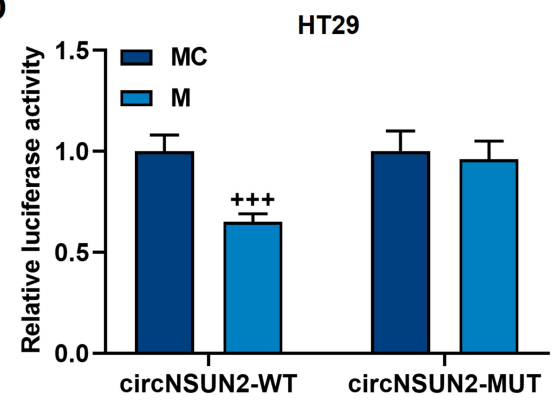

Figure 3 Among the differentially expressed circRNAs in CRC, only circNSUN2 not only had a target site for miR-296-5p, but also could be regulated by ALO. (A) qRT-PCR detected the regulation of $0.8 \mathrm{mmol} / \mathrm{L}$ ALO on the differentially expressed circRNAs in CRC. GAPDH played the role of internal reference. (B) The TargetScan database (http:// www.targetscan.org) was used to predict the target sites of circNSUN2 and miR-296-5p. (C and D) The dual luciferase experiment confirmed that circNSUN2 can bind to miR296-5p in SW480 and HT29 cells. All experiments were repeated three times to obtain average values. ${ }^{* * *} p<0.00$ I vs Control; ${ }^{+++} p<0.00$ I vs $\mathrm{MC}$.

Abbreviation: MC, miR-296-5p mimic control.

CRC cells were significantly inhibited; on the contrary, the number of apoptotic cancer cells was significantly increased. This is consistent with the previous experimental results. ${ }^{21}$ In order to probe deeper into the upstream mechanism of ALO in alleviating CRC carcinogenesis through the miR-296-5p/STAT3 axis, we screened and verified the upstream targeting circRNA of miR-296-5p.

CircRNAs are a type of covalently closed circular noncoding RNA formed by back-splicing of mRNA precursors (pre-mRNA). ${ }^{22}$ CircRNA was considered to be a useless splicing by-product in early research. With the deepening of research, it is found that circRNA has a wide range of sources and plays a variety of functional roles in the growth and development of organisms, with the characteristics of conservative, stable, and tissue-specific. ${ }^{22,23}$ The most familiar and extensively studied function of circRNA is its sponging effect on miRNA. ${ }^{24}$ For example, circRNA ciRS-7 which contains more than 70 miR-7 binding sites can achieve competitive adsorption of miR7 through the AGO2 protein. ${ }^{25}$ Besides, increasing reports on cancer indicate that circRNAs, such as circRNAcTFRC, circPSMC3 and circSETD3, act as sponges of miRNAs and participate in transcriptional regulation. ${ }^{26-28}$
Based on these literature reports, we screened circRNAs that were reported to be abnormally expressed in CRC, and finally obtained 12 circRNAs. After detecting their expressions in ALO-treated cancer cells and predicting their binding sequence for miR-296-5p, circNSUN2 was finally identified as the research object of this study.

CircNSUN2, which maps to the $5 \mathrm{p} 15$ amplicon in CRCs, was confirmed by Chen et al to regulate cytoplasmic output and promote liver metastasis of CRC through N 6-methyladenosine modification. ${ }^{29}$ Similarly, our experimental results uncovered that knockdown of circNSUN2 inhibited proliferation and accelerated apoptosis in CRC cells. More importantly, we for the first time revealed that ALO can prevent the activation of circNSUN2 and offset the promotion effect of overexpression of circNSUN2 on esophageal cancer progression. Since circNSUN2 and miR-296-5p have targeting sequences, we verified the cellular regulatory effects of circNSUN2 and miR-296-5p/STAT3 through dual luciferase experiments and rescue experiments. The final result confirmed our conjecture that regulating the circNSUN2/ miR-296-5p/STAT3 axis can reduce the proliferation rate and increase the apoptosis rate of CRC cells. 

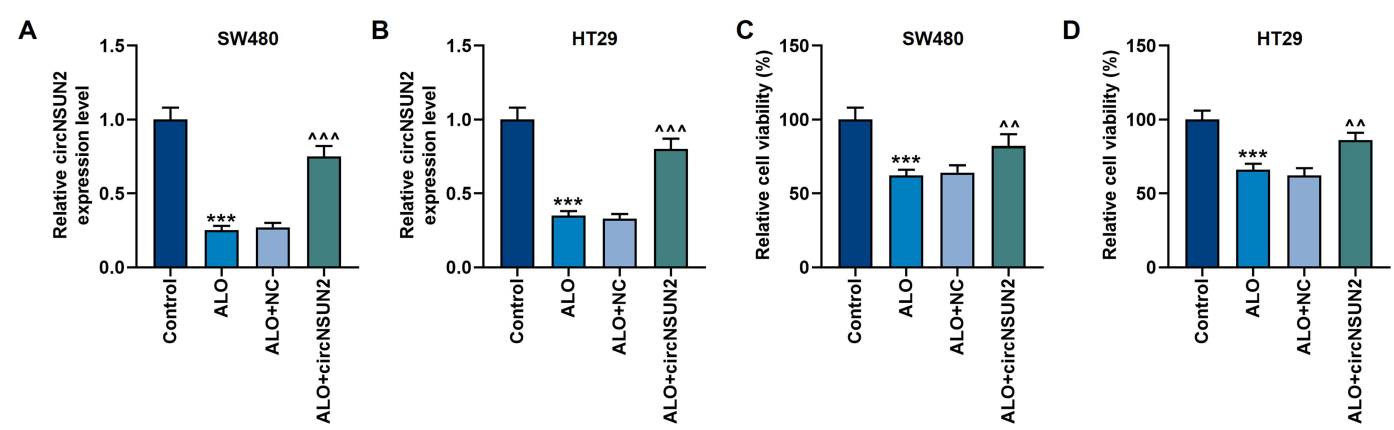

$\mathbf{E}$
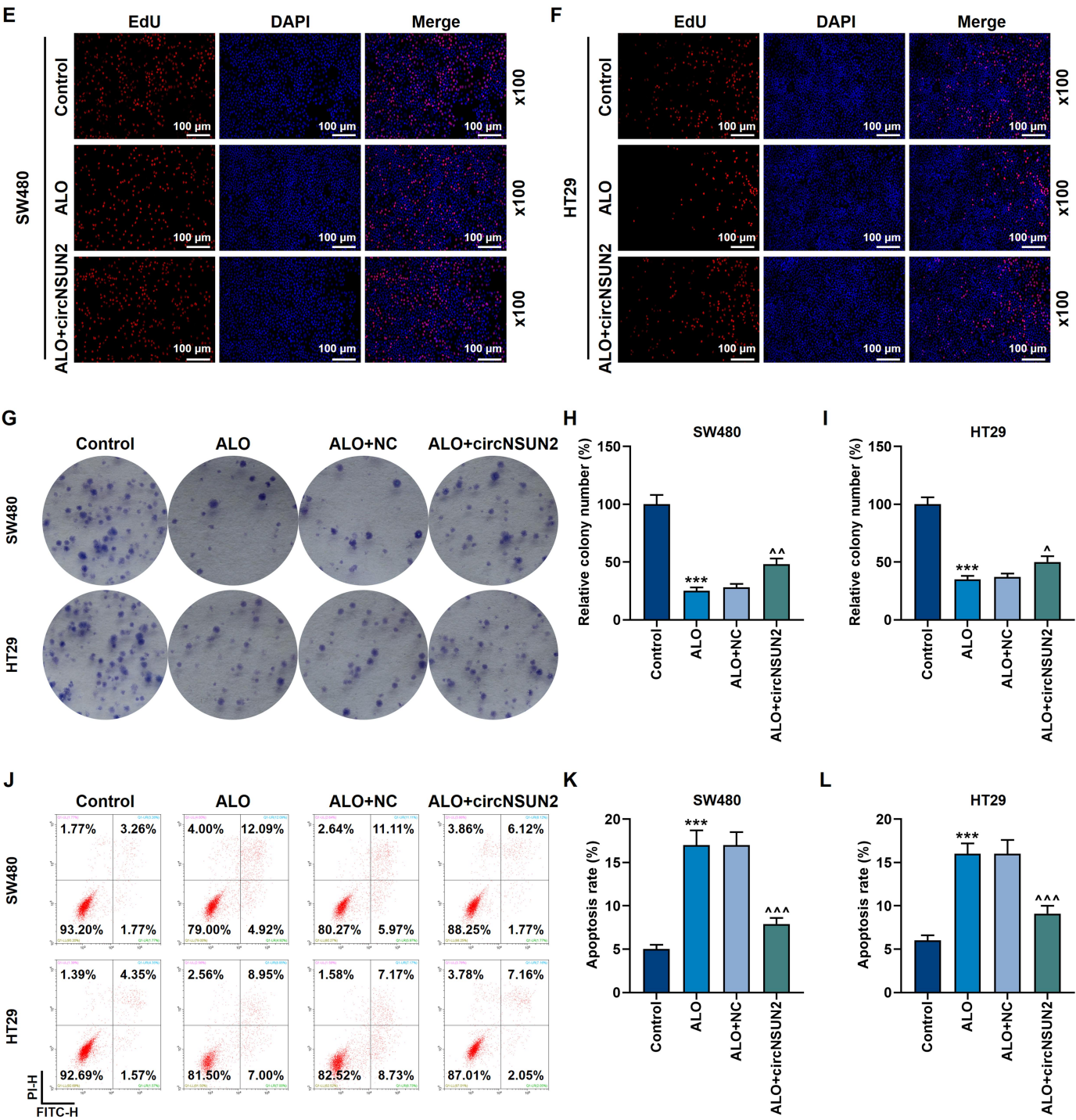

Figure 4 Overexpression of circNSUN2 partially offset the suppression of ALO on the biological behavior of cancer cells. (A and B) The qRT-PCR experiment showed that ALO $(0.8 \mathrm{mmol} / \mathrm{L})$ inhibited the expression of circNSUN2 in SW480 and HT29 cells. GAPDH played the role of internal reference. (C and D) The MTT experiment showed that overexpression of circNSUN2 partially offset the inhibition of ALO on cell viability in SW480 and HT29 cells. (E and F) EdU cell proliferation experiments showed that overexpression of circNSUN2 partially offset the inhibition of ALO on proliferation in SW480 and HT29 cells. The magnification was I00x. (G-I) The clone formation experiment showed that overexpression of circNSUN2 partially offset the inhibition of ALO on proliferation in SW480 and HT29 cells. (J-L) Flow cytometry experiments showed that overexpression of circNSUN2 partially offset the promotion of ALO on apoptosis in SW480 and HT29 cells. All experiments were repeated three times to obtain average values. ${ }^{* * *} p<0.001$ vs Control; ${ }^{\wedge} p<0.05,{ }^{\wedge} p<0.01,{ }^{\wedge \wedge} p<0.001$ vs ALO+NC.

Abbreviations: CircNSUN2, NOP2/Sun RNA methyltransferase 2; NC, negative control. 


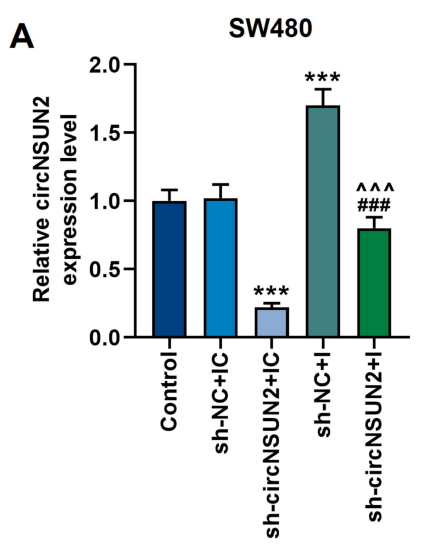

E

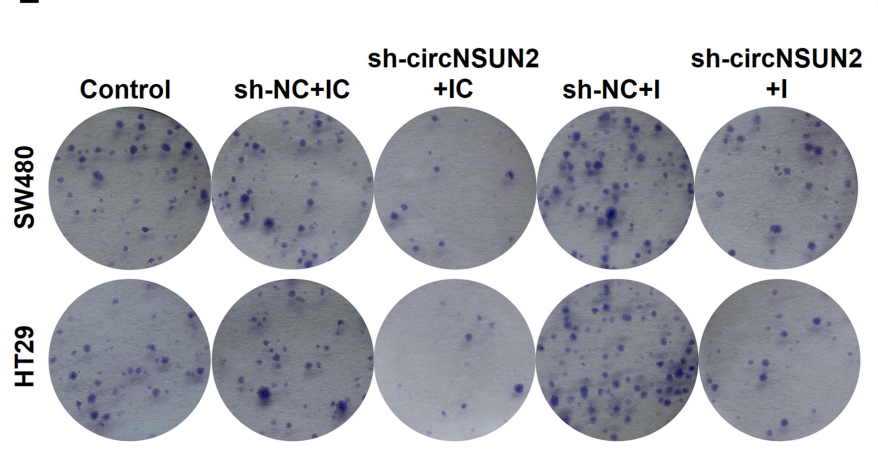

H

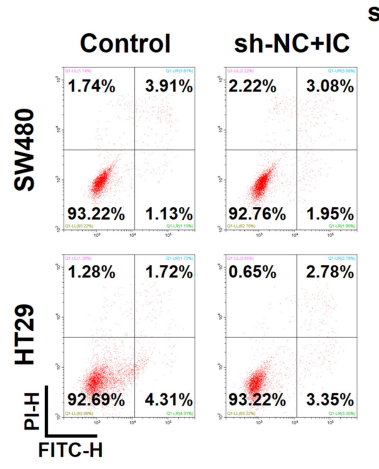

B

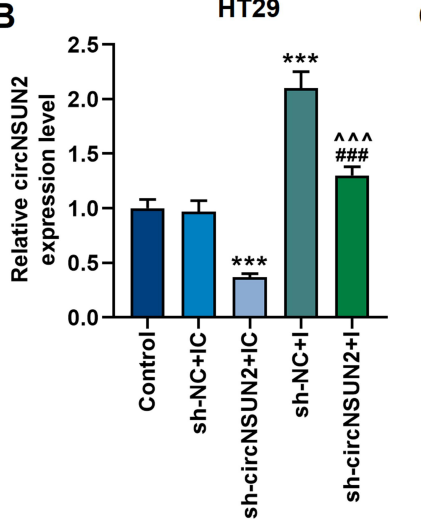

sh-circNSUN2 sh-circNSUN2
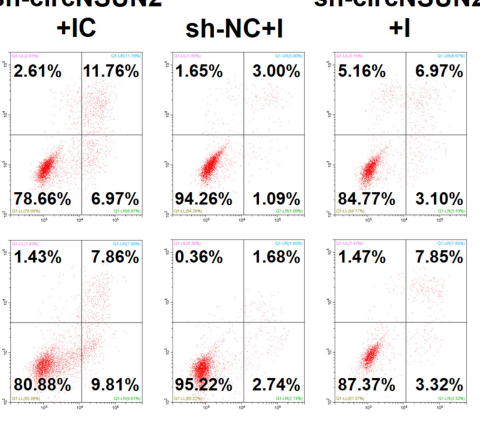

C

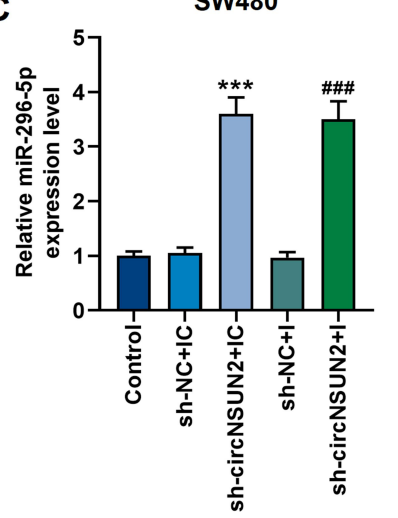

$\mathbf{F}$

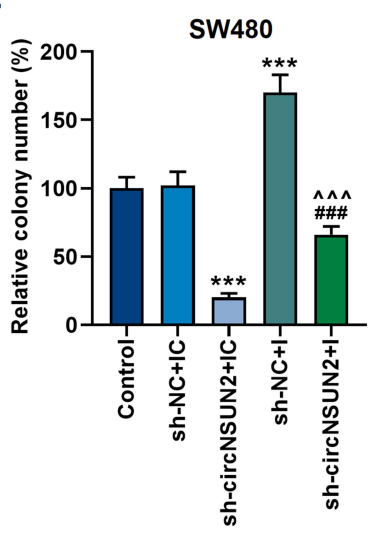

D

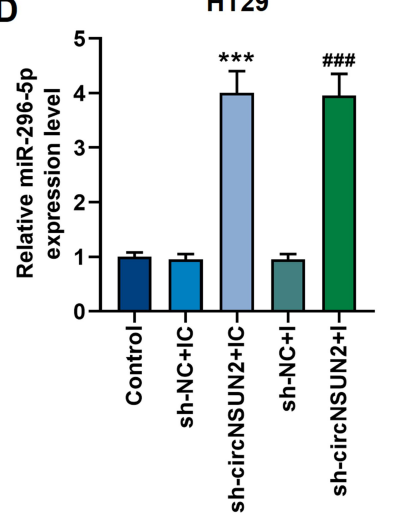

G

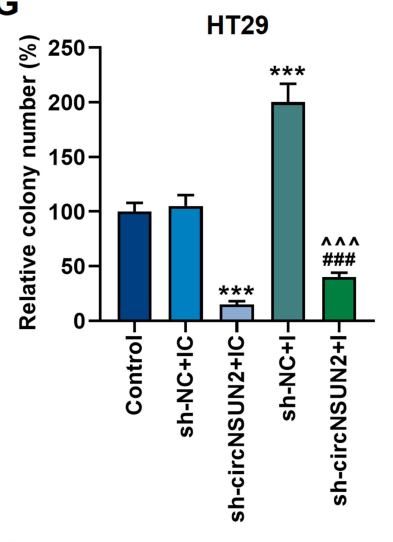

I

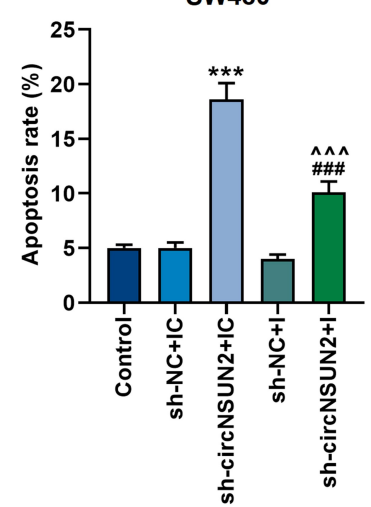

J

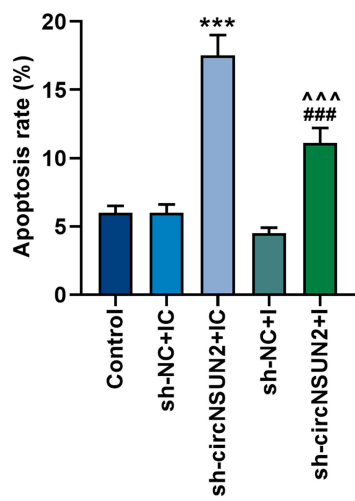

Figure 5 Sh-circNSUN2 inhibited the malignant development of CRC cells, which was neutralized by miR-296-5p inhibitor. (A and B) The qRT-PCR experiment showed that miR-296-5p inhibitor neutralized the inhibitory effect of sh-circNSUN2 on circNSUN2 expression. GAPDH played the role of internal reference. (C and D) The qRTPCR experiment showed that sh-circNSUN2 up-regulated the mRNA level of miR-296-5p. U6 played the role of internal reference. (E-G) The clone formation experiment showed that the effect of sh-circNSUN2 on the proliferation of SW480 and HT29 cells was neutralized by miR-296-5p inhibitor. (H-J) Flow cytometry experiments showed that the effect of sh-circNSUN2 on promoting the apoptosis of SW480 and HT29 cells was neutralized by miR-296-5p inhibitor. All experiments were repeated three times

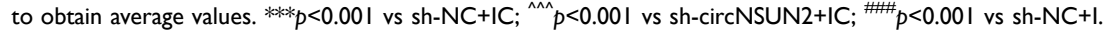

Abbreviations: Sh-circNSUN2, silent circNSUN2; sh-NC, silent negative control; IC, miR-296-5p inhibitor control; I, miR-296-5p inhibitor.

At present, blocking proliferation and increasing apoptosis in cancer cells are the intensively discussed mechanisms in CRC study. Tumor cells can proliferate quickly and unrestrictedly, and thus, inhibiting their proliferation can produce an anti-tumor effect. ${ }^{30}$ Apoptosis, alternatively called programmed cell death, is an autonomous cell death process strictly controlled by multiple genes. Apoptosis is an important part of cell life cycle, and it is also an important link in regulating the development of the body and maintaining the stability of the internal environment in organisms. ${ }^{31,32}$ Our research shows that ALO inhibits the proliferation and promotes the apoptosis of CRC cells by regulating the circNSUN2/miR-296-5p/STAT3 pathway, and ultimately prevents the tumorigenesis of CRC. 
A

\begin{tabular}{|l|l|}
\hline STAT3-WT & Target: 5' CAUGAUGCACACUAUGGGGCCCC 3' \\
hsa-miR-296-5p & miRNA : $3{ }^{\prime}$ UGUCCUAACUCCCCCCCGGGA $5{ }^{\prime}$ \\
STAT3-MUT & Target: 5 ' CAUGAUGCACACUAUGAGUCGCC 3' \\
\hline
\end{tabular}

B

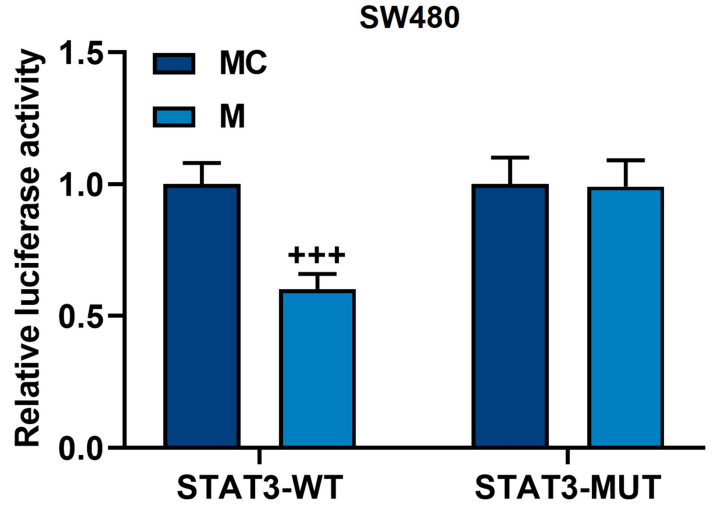

C

HT29

Figure 6 The targeted binding of miR-296-5p to STAT3. (A) TargetScan was used to predict the binding sequence of miR-296-5p and STAT3. (B and C) Dual luciferase experiment confirmed that miR-296-5p can bind to STAT3 in SW480 and HT29 cells. All experiments were repeated three times to obtain average values. ${ }^{+++} p<0.00 \mathrm{I}$ vs MC.

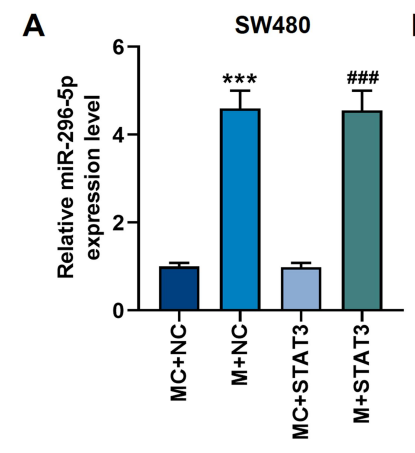

E

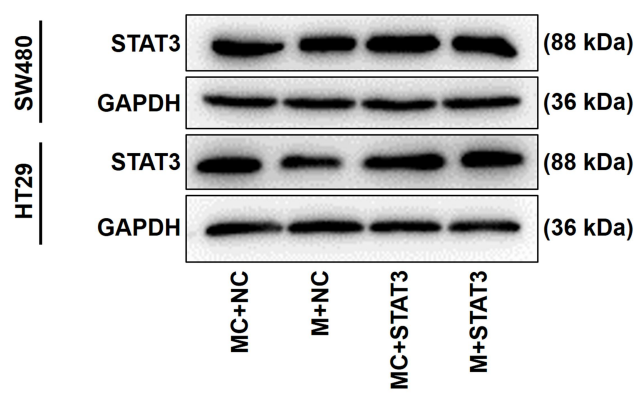

B

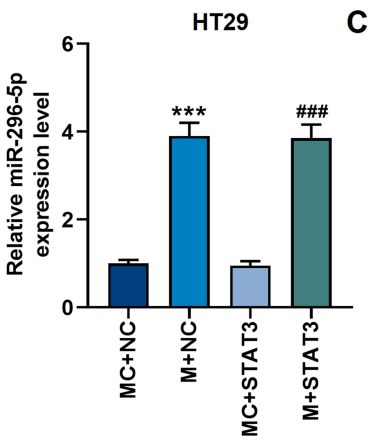

$F$

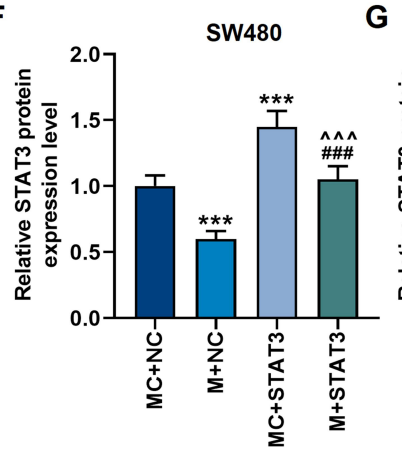

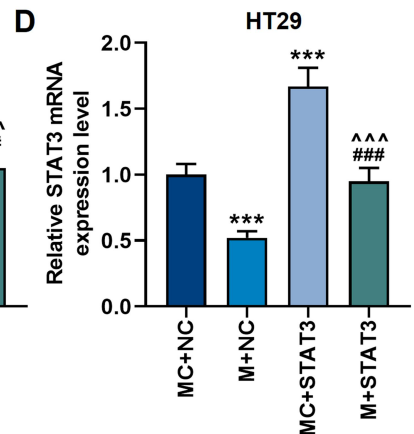

G

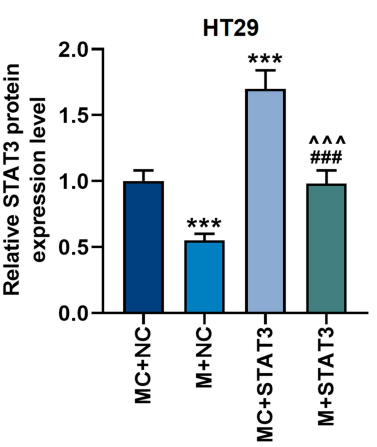

Figure 7 Up-regulation of miR-296-5p inhibited the expression of STAT3 in CRC cells. (A and B) The qRT-PCR experiment showed that miR-296-5p mimic up-regulated the mRNA level of miR-296-5p. U6 played the role of internal reference. (C and D) The qRT-PCR experiment showed that miR-296-5p mimic suppressed the mRNA level of STAT3, while overexpression of STAT3 reversed this effect. GAPDH played the role of internal reference. (E-G) The Western blot experiment showed that miR-296-5p mimic inhibited the protein level of STAT3, while over-expression of STAT3 reversed this effect. GAPDH played the role of internal reference. All experiments were repeated three times to obtain average values. ${ }_{* * * *} p<0.00$ I vs $\mathrm{MC}+\mathrm{NC} ;{ }^{\wedge \wedge} p<0.00 \mathrm{I}$ vs $\mathrm{M}+\mathrm{NC}$; ${ }^{\prime \prime \prime} p<0.00$ I vs MC+STAT3.

Abbreviation: STAT3, signal transducer and activator of transcription 3. 

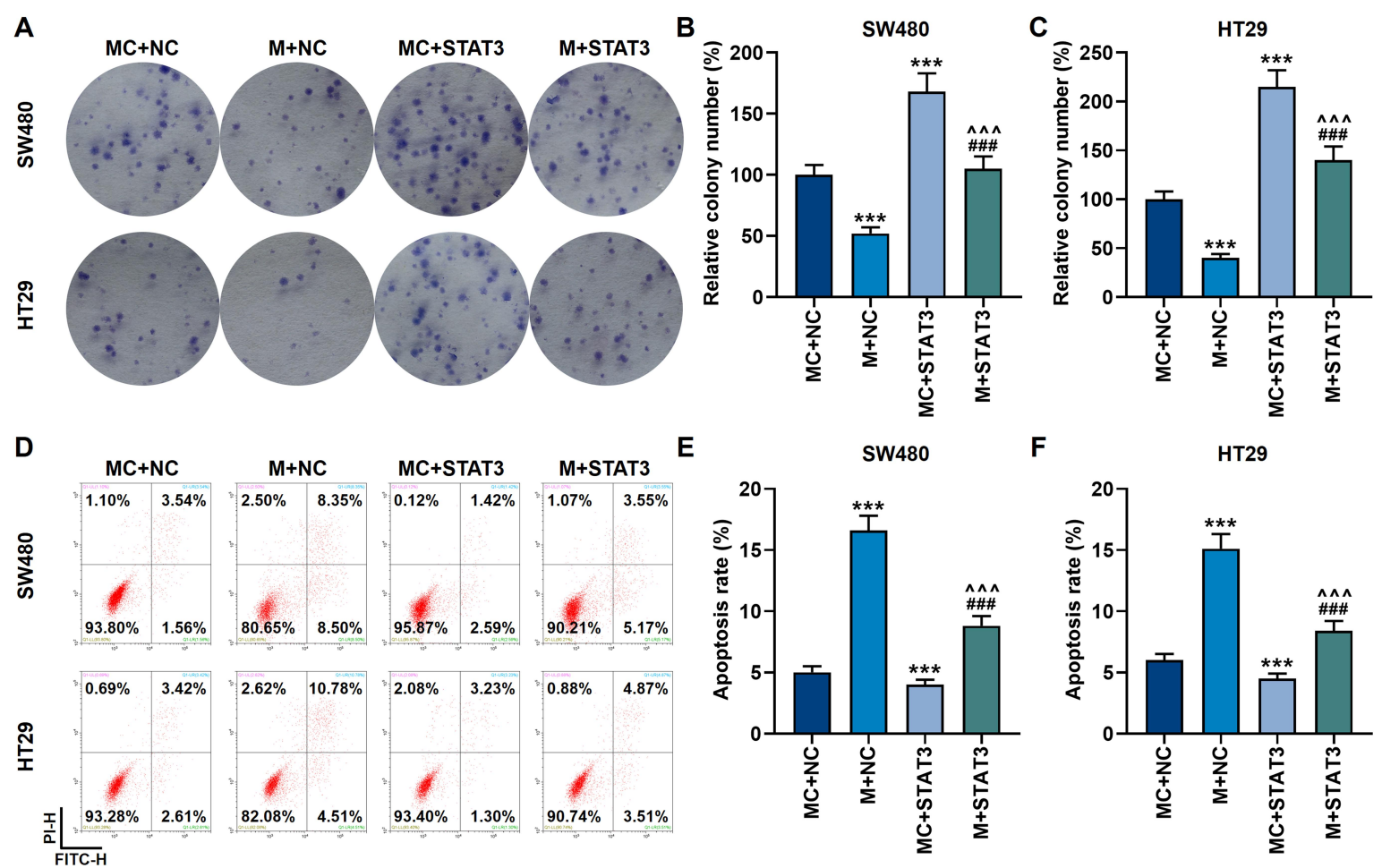

E

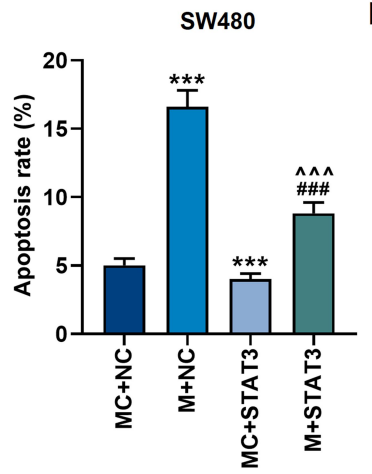

$\mathbf{F}$

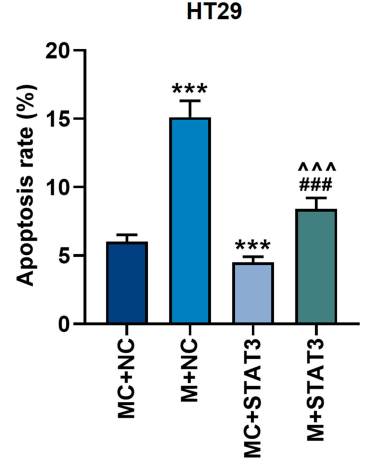

G

H
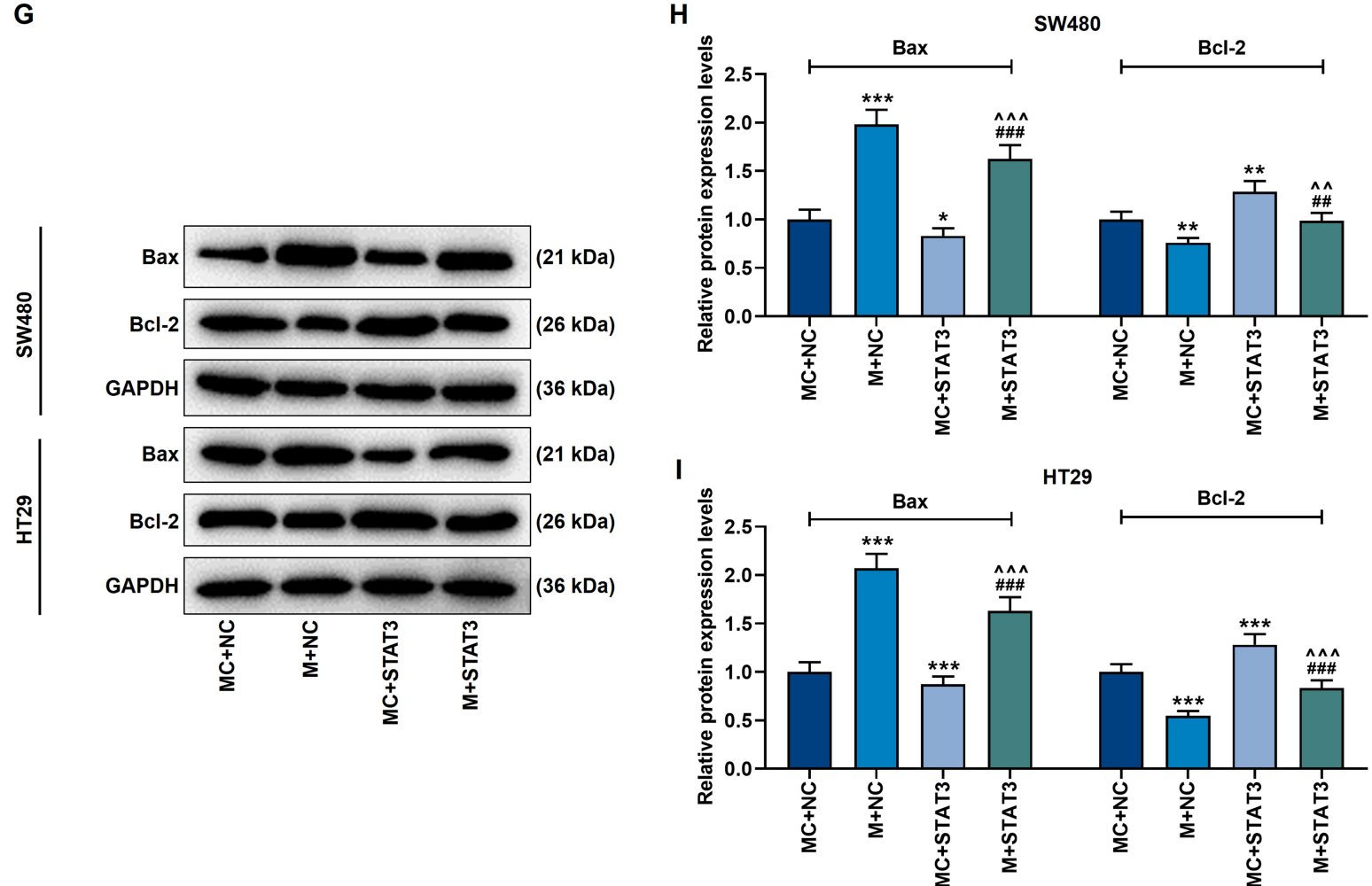

Figure 8 MiR-296-5p mimic inhibited proliferation and promoted apoptosis in CRC cells by regulating apoptosis-related genes, which was partially reversed by overexpression of STAT3. (A-C) The clone formation experiment showed that the inhibitory effect of miR-296-5p mimic on the proliferation of SW480 and HT29 cells was neutralized by overexpressed STAT3. (D-F) Flow cytometry experiments showed that miR-296-5p mimic promoted the apoptosis of SW480 and HT29 cells, which was neutralized by overexpressed STAT3. (G-I) Western blot experiments showed that the regulation of apoptosis-related protein expression by miR-296-5p mimic was neutralized by overexpressed STAT3. GAPDH played the role of internal reference. All experiments were repeated three times to obtain average values. ${ }^{*} p<0.05$, $* * p<0.0 \mathrm{I}$, $* * * p<0.001$ vs MC+NC; ${ }^{\wedge} p<0.01,{ }^{\wedge \wedge} p<0.001$ vs $\mathrm{M}+\mathrm{NC} ;{ }^{\#} p<0.01,{ }^{\# \#} p<0.001$ vs MC+STAT3. 
There are still certain limitations in our research. Although we have confirmed the effect of ALO on inducing apoptosis and reducing proliferation in CRC cells and clarified the underlying mechanism, the circNSUN2/miR296-5p/STAT3 axis and the pathways related to proliferation and apoptosis have not been analyzed yet, which will be further explored in future research. In addition, the anticancer effect of ALO on CRC and its experimental concentration needs to be further tested in animal and clinical experiments. Whether ALO has an impact on drug resistance in $\mathrm{CRC}$ is also a direction of future research.

\section{Disclosure}

The authors declare no conflicts of interest in this work.

\section{References}

1. Bray F, Ferlay J, Soerjomataram I, Siegel RL, Torre LA, Jemal A. Global cancer statistics 2018: GLOBOCAN estimates of incidence and mortality worldwide for 36 cancers in 185 countries. CA Cancer J Clin. 2018;68(6):394-424. doi:10.3322/caac.21492

2. Brody H. Colorectal cancer. Nature. 2015;521(7551):S1. doi:10.1038/521S1a

3. Dekker E, Tanis PJ, Vleugels JLA, Kasi PM, Wallace MB. Colorectal cancer. Lancet. 2019;394(10207):1467-1480. doi:10.1016/s01406736(19)32319-0

4. The Lancet O. Colorectal cancer: a disease of the young? Lancet Oncol. 2017;18(4):413. doi:10.1016/s1470-2045(17)30202-4

5. Patel SG, Ahnen DJ. Colorectal cancer in the young. Curr Gastroenterol Rep. 2018;20(4):15. doi:10.1007/s11894-018-0618-9

6. Costas-Chavarri A, Nandakumar G, Temin S, et al. Treatment of patients with early-stage colorectal cancer: ASCO resource-stratified guideline. J Glob Oncol. 2019;5(5):1-19. doi:10.1200/jgo.18.00214

7. Zellweger M, Abdelnour-Berchtold E, Krueger T, Ris HB, Perentes JY, Gonzalez M. Surgical treatment of pulmonary metastasis in colorectal cancer patients: current practice and results. Crit Rev Oncol Hematol. 2018;127:105-116. doi:10.1016/j.critrevonc.20 18.05.001

8. Chakedis J, Schmidt CR. Surgical treatment of metastatic colorectal cancer. Surg Oncol Clin N Am. 2018;27(2):377-399. doi:10.1016/j. soc. 2017.11 .010

9. Kim JH. Chemotherapy for colorectal cancer in the elderly. World $J$ Gastroenterol. 2015;21(17):5158-5166. doi:10.3748/wjg.v21. i17.5158

10. Sileri P, D’Ugo S, Benavoli D, et al. Metachronous splenic metastasis from colonic carcinoma five years after surgery: a case report and literature review. South Med J. 2009;102(7):733-735. doi:10.1097/ SMJ.0b013e3181a93c39

11. Koi M, Carethers JM. The colorectal cancer immune microenvironment and approach to immunotherapies. Future Oncol. 2017;13 (18):1633-1647. doi:10.2217/fon-2017-0145

12. Wrobel P, Ahmed S. Current status of immunotherapy in metastatic colorectal cancer. Int $J$ Colorectal Dis. 2019;34(1):13-25. doi:10.1007/s00384-018-3202-8

13. Fu X, Sun F, Wang F, et al. Aloperine protects mice against DSS-induced colitis by PP2A-mediated PI3K/Akt/mTOR signaling suppression. Mediators Inflamm. 2017;2017:5706152. doi:10.1155/ $2017 / 5706152$
14. Tolkachev ON, Monakhova TE, Sheichenko VI, Kabanov VS, Fesenko OG, Proskurnina NF. Alkaloids of a new type from Sophora alopecuroides L. Chem Nat Compd. 1975;11(1):29-34. doi: $10.1007 / \mathrm{BF} 00567025$

15. Yu HI, Shen HC, Chen SH, et al. Autophagy modulation in human thyroid cancer cells following aloperine treatment. Int $J$ Mol Sci. 2019;20(21):5315. doi:10.3390/ijms20215315

16. Liu JS, Huo CY, Cao HH, et al. Aloperine induces apoptosis and G2/ $\mathrm{M}$ cell cycle arrest in hepatocellular carcinoma cells through the PI3K/Akt signaling pathway. Phytomedicine. 2019;61:152843. doi:10.1016/j.phymed.2019.152843

17. Ling Z, Guan H, You Z, et al. Aloperine executes antitumor effects through the induction of apoptosis and cell cycle arrest in prostate cancer in vitro and in vivo. Onco Targets Ther. 2018;11:2735-2743. doi: $10.2147 /$ ott.s 165262

18. Tian D, Li Y, Li X, Tian Z. Aloperine inhibits proliferation, migration and invasion and induces apoptosis by blocking the Ras signaling pathway in human breast cancer cells. Mol Med Rep. 2018;18 (4):3699-3710. doi:10.3892/mmr.2018.9419

19. Ciesielska A, Stączek P. Selection and validation of reference genes for qRT-PCR analysis of gene expression in microsporum canis growing under different adhesion-inducing conditions. Sci Rep. 2018;8(1):1197. doi:10.1038/s41598-018-19680-9

20. Müller MF, Ibrahim AE, Arends MJ. Molecular pathological classification of colorectal cancer. Virchows Arch. 2016;469(2):125-134. doi:10.1007/s00428-016-1956-3

21. Zhang L, Zheng Y, Deng H, Liang L, Peng J. Aloperine induces G2/ $\mathrm{M}$ phase cell cycle arrest and apoptosis in HCT116 human colon cancer cells. Int J Mol Med. 2014;33(6):1613-1620. doi:10.3892/ ijmm.2014.1718

22. Chen LL, Yang L. Regulation of circRNA biogenesis. RNA Biol. 2015;12(4):381-388. doi:10.1080/15476286.2015.1020271

23. Li S, Teng S, Xu J, et al. Microarray is an efficient tool for circRNA profiling. Brief Bioinform. 2019;20(4):1420-1433. doi:10.1093/bib/ bby006

24. Conn SJ, Pillman KA, Toubia J, et al. The RNA binding protein quaking regulates formation of circRNAs. Cell. 2015;160 (6):1125-1134. doi:10.1016/j.cell.2015.02.014

25. Hansen TB, Jensen TI, Clausen BH, et al. Natural RNA circles function as efficient microRNA sponges. Nature. 2013;495 (7441):384-388. doi:10.1038/nature11993

26. Xu L, Feng X, Hao X, et al. CircSETD3 (Hsa_circ_0000567) acts as a sponge for microRNA-421 inhibiting hepatocellular carcinoma growth. J Exp Clin Cancer Res. 2019;38(1):98. doi:10.1186/s13046019-1041-2

27. Ye CY, Chen L, Liu C, Zhu QH, Fan L. Widespread noncoding circular RNAs in plants. New Phytol. 2015;208(1):88-95. doi:10.1111/nph.13585

28. Wang Y, Yang M, Wei S, Qin F, Zhao H, Suo B. Identification of circular RNAs and their targets in leaves of triticum aestivum 1. under dehydration stress. Front Plant Sci. 2016;7:2024. doi:10.3389/ fpls.2016.02024

29. Chen RX, Chen X, Xia LP, et al. N(6)-methyladenosine modification of circNSUN2 facilitates cytoplasmic export and stabilizes HMGA2 to promote colorectal liver metastasis. Nat Commun. 2019;10 (1):4695. doi:10.1038/s41467-019-12651-2

30. Fletcher JS, Springer MG, Choi K, et al. STAT3 inhibition reduces macrophage number and tumor growth in neurofibroma. Oncogene. 2019;38(15):2876-2884. doi:10.1038/s41388-018-0600-x

31. Mohammad RM, Muqbil I, Lowe L, et al. Broad targeting of resistance to apoptosis in cancer. Semin Cancer Biol. 2015;35:S78-s103. doi:10.1016/j.semcancer.2015.03.001

32. Goldar S, Khaniani MS, Derakhshan SM, Baradaran B. Molecular mechanisms of apoptosis and roles in cancer development and treatment. Asian Pac J Cancer Prev. 2015;16(6):2129-2144. doi:10.7314/apjcp.2015.16.6.2129 


\section{Publish your work in this journal}

Drug Design, Development and Therapy is an international, peerreviewed open-access journal that spans the spectrum of drug design and development through to clinical applications. Clinical outcomes, patient safety, and programs for the development and effective, safe, and sustained use of medicines are a feature of the journal, which has also been accepted for indexing on PubMed Central. The manuscript management system is completely online and includes a very quick and fair peer-review system, which is all easy to use. Visit http://www. dovepress.com/testimonials.php to read real quotes from published authors. 